Published in final edited form as:

Surg Endosc. 2012 April ; 26(4): 1128-1134. doi:10.1007/s00464-011-2011-8.

\title{
Effect of Haptic Feedback in Laparoscopic Surgery Skill Acquisition
}

\author{
M. Zhou^, S. Tse ${ }^{\star}$, A. Derevianko^, D.B. Jones^, S.D. Schwaitzberg\#, and C. G. L. Cao \\ ${ }^{*}$ Mechanical Engineering Department, Tufts University, Medford, MA \\ ${ }^{\wedge}$ Minimally Invasive Surgery Division, Beth Israel Deaconess Medical Center, Boston, MA \\ \#Surgery, Cambridge Health Alliance Hospital, Cambridge, MA
}

\section{Abstract}

Background-The benefits of haptic feedback in laparoscopic surgery training simulators is a topic of debate in the literature. It is hypothesized that novice surgeons may not benefit from the haptic information, especially during the initial phase of learning a new task. Therefore, providing haptic feedback to novice trainees in the early stage of training may be distracting and detrimental to learning.

Objective-A controlled experiment was conducted to examine the effect of haptic feedback on the learning curve of a complex laparoscopic suturing and knot-tying task.

Method-The ProMIS and the MIST-VR surgical simulators were used to represent conditions with and without haptic feedback, respectively. Twenty novice subjects (10 per simulator) were trained to perform suturing and knot-tying and practiced the tasks over eighteen one-hour sessions.

Results-At the end of the 3-week training period, subjects performed equally fast but more consistently with haptics (ProMIS) than without (MIST-VR). Subjects showed slightly higher learning rate and reached the first plateau of the learning curve earlier with haptic feedback.

Conclusion-In general, learning with haptic feedback was significantly better than without haptic feedback for a laparoscopic suturing and knot-tying task, but only in the first 5 hours of training.

Application-Haptic feedback may not be warranted in laparoscopic surgical trainers. The benefits of a shorter time to the first performance plateau and more consistent initial performance should be balanced with the cost of implementing haptic feedback in surgical simulators.

Please send correspondence to: Caroline GL Cao, Tufts University, Department of Mechanical Engineering, 200 College Avenue, Medford, MA 02155, Phone: 617-627-2484, Fax: 617-627-3058, caroline.cao@tufts.edu.

NOTE: Preliminary results of this study were presented at the $52^{\text {nd }}$ Annual Meeting of the Human Factors and Ergonomics Society, September 22-26, 2008, New York, NY., and the 2008 meeting of the Society of American Gastrointestinal and Endoscopic Surgeons, Philadelphia, PA, April 9-12, 2008.

Financial Disclosures

Dr. M. Zhou, and Ms. S. Tse, have no conflicts of interest or financial ties to disclose.

Dr. A. Derevianko has consulted with Elsevier, Inc., and the Medical Education Advisory Board.

Dr. D.B. Jones has consulted with Stryker Endoscopy, Olympus, research with Allergan, Coviden, and institutional training grants from Covidien and Ethicon Endosurgery. He has no conflicts of interest with this manuscript.

Dr. S.D. Schwaitzberg has consulted for MMDI, Endocore, and Olympus, and has served on the advisory committee for MITI,

Stryker, Surgiquest, Cambridge Endo, Neatstitch, and Acuity Bio. He has no conflicts of interest with this manuscript.

Dr. C.G.L. Cao has consulted for St. Jude Medical. She has no conflicts of interest with this manuscript. 


\section{Keywords}

Haptic Feedback; Laparoscopic Surgery; Skill Acquisition; Learning Curve

Laparoscopic surgery has very important advantages over open surgery in that it minimizes tissue trauma, shortens patient recovery time, reduces the length of hospital stay, and hence health care costs. It is a preferred alternative to open surgery in many procedures. However, it presents considerable challenges for surgeons, such as a limited view of the surgical field, lost of depth perception due to a 2D visual display, the fulcrum effect in tool manipulation, and distorted haptic feedback due to mediation by long tools and the masking rubber seal in trocars $[1,2]$. A great deal of training is required to adapt to these limitations in laparoscopic surgery.

Recently, a transformation in the approach to surgical training has taken place, with technological innovation such as surgical simulators and virtual reality simulation playing an increasingly important role [3, 4], for both practical and ethical reasons. However, the fidelity of simulation remains a technical challenge for the engineering community, and the use of simulators as training tools an empirical question for surgical educators. Although low-fidelity simulators have been shown to provide significant skill transfer to the operating room $[3,5]$, it is generally considered that realistic simulations with multisensory feedback that includes haptic feedback could provide a better learning experience and thus better outcomes [6].

The role of haptic feedback is of special interest in surgery because it is critical in the discrimination of healthy versus abnormal tissues, identification of organs, and motor control. Haptic perception is greatly impaired in laparoscopic surgery as exploration is done through a rigid probe [7-13]. Expert surgeons agree that haptic feedback is a necessity in surgery, and training on the box trainer is preferable over virtual reality simulator systems for laparoscopic suturing because VR simulators lack realism and haptic feedback [14]. Force feedback can improve robot-assisted knot-tying with fine suture [15], reduce the overall forces applied and the number of accidental incursions into sensitive structures, as well as faster task completion time, and the straightness of suturing [16]. However, force feedback did not improve the rate and precision of dissection [17].

Several studies have investigated the impact of haptic feedback in surgical training, with mixed results. One study showed that early exposure to haptic feedback enhanced performance in a diathermy task [18]. Others have shown that the learning curve based on the speed of task completion reached a plateau after three trials while performance accuracy did not stabilize after ten trials on a box trainer [19]. For an intracorporeal knot-tying task, significant improvement in performance was observed after one trial, with further significant improvement at the end of ten trials [20]. Two other learning curve studies where trainees used the virtual reality trainer, MIST-VR, showed that performance was significantly improved after a 5-day training period [21] and that performance variables reached a plateau by trial 5 [22]. However, for a knot-tying task, there was no significant differences in performance between the MIST-VR and box trainer on performance time for any trial [23].

A review of aviation training literature [24] suggests that irrelevant stimuli in a high-fidelity simulator can make task learning more difficult as the novice trainee had to learn to ignore these stimuli. As current surgical training simulators are low in fidelity with respect to their visual and task representation (i.e., using peas or graphical spheres to represent tissue), the addition of haptic feedback may be distracting for the novice trainee [25]. Since novices in surgery are the major target group for training in simulators [26], as simulators seem to be 
most useful for the early part of the learning curve [27], we need to know the benefits of haptic feedback in the early stage of training.

We hypothesized that 1) subjects who trained with haptic feedback would perform better throughout the learning phase than those trained without haptic feedback, and 2) trainees who trained with haptic feedback would reach the first plateau in the learning curve earlier and have a higher learning rate than those trained without haptic feedback.

\section{Methods \\ Participants}

Twenty graduate and undergraduates ( 6 females and 14 males) without any prior laparoscopic experience participated in this study. Nineteen of the subjects were righthanded, and one ambidextrous. Subjects were aged between 21 and 34 years old.

\section{Apparatus}

Two surgical simulators, MIST-VR and ProMIS, were used in this experiment (Figure 1). MIST-VR is a virtual reality simulator that does not provide haptic feedback, while ProMIS is a physical simulator that provides haptic feedback similar to that experienced in surgery. MIST-VR consists of a computer, monitor, and a laparoscopic tool base. ProMIS consists of a computer, monitor, laparoscopic tools, and life-size model of the upper torso with a light source and three built-in cameras.

\section{Procedure}

The same laparoscopic suturing and knot-tying task was performed on the MIST-VR and ProMIS. The task involved making two single loop half-square knots, forming a complete square knot, using two needle drivers. In the no haptics condition, the subjects sutured a virtual organ on MIST-VR system. While in the haptics condition, the subjects sutured a Penrose drain velcroed to a block on the ProMIS system. Both the virtual organ and Penrose drain had pre-marked entry and exit points for the suture.

Prior to starting the study, all subjects received a one -hour orientation, which included a brief introduction to laparoscopic surgery and a demonstration of both open and laparoscopic suturing by an expert surgeon. After the orientation, subjects practiced the task one hour a day, six days a week, over three consecutive weeks for a total of 18 training sessions. During the hour-long training sessions, subjects attempted as many knots as possible. Further instruction was given to the subjects if they appeared to be struggling with the task. Moreover, knowledge of performance results were shown to the subjects at the end of each trial. On the ProMIS simulator, these results included time to task completion, instrument path, and instrument smoothness; on the MIST-VR, results shown included time to task completion, errors, and overall score.

\section{Experimental Design and Data Collected}

Subjects were randomly assigned to one of two conditions: Haptics, and No-Haptics. There were ten subjects for each condition. Those in the Haptics condition performed the task using the ProMIS simulator, while those in the No-Haptics condition used the MIST-VR simulator. The performance measure was the time to complete one square knot successfully. Unsuccessful knots were not counted. A successful suture is a complete square knot (two half-square knots that alternate in direction) on the designated entry and exit points. 


\section{Data Analysis}

Individual learning curves were obtained from the time to task completion measure for each subject. One-way ANOVA was performed within each group to examine the effect of training sessions. In addition, the differences between the first session and all subsequent sessions, and between the last session and all prior sessions were compared using a post-hoc Scheffe test.

The learning rate was calculated according to the equation [28]:

$$
\mathrm{Y}_{\mathrm{x}}=\mathrm{KX}^{\mathrm{N}}
$$

Learning rate: $2^{\mathrm{N}}$

$\mathrm{Y}_{\mathrm{X}}$ : production time for $\mathrm{X}$ th unit in sequence

$\mathrm{K}$ : time required for first unit

$\mathrm{N}$ : exponent leading to learning rate $\left(2^{\mathrm{N}}\right)$

$\mathrm{X}$ : number of production units

One-way ANOVA was performed to compare the learning rates in two groups. Paired $t$-tests were performed to compare the time to task completion, variance of task completion time per session, and the best performance time of the session for the two groups. The time to task completion for each suture and between subjects variance in the training were also analyzed using paired $t$-test.

\section{Results}

\section{Learning Curve}

Individual learning curves were plotted using each subject's average time to task completion per session from session 1 to session 18 (Figure 2). Averaged suturing and knot-tying times for the first and last (18th) sessions are shown in Table 1. Performance session showed significant main effects for both groups (Haptics group, $F(17,1226)=38.8, p<.001$; NoHaptics group, $F(17,2737)=65.2, p<.001)$. There were significant differences between performance in session 1 and performance in all subsequent sessions in each group $(\mathrm{p}<$. 001), suggesting that learning was occurring in the first session. The performance differences between a given session and the last session are also shown in Table 1.

There were significant performance differences between sessions 1-5 and the last session (session 18) in the No-Haptics group, while significant differences in performance were found only between sessions 1-4 and the last session in the Haptics group. That is, the NoHaptics group reached a performance plateau by the 6th session, while the Haptics group reached the plateau earlier, by the 5 th session.

When comparing the learning rate between the Haptics and No-Haptics group, results from the one-way ANOVA showed no significant differences overall, $\mathrm{F}(1,18)=4.02, \mathrm{p}<.061$. The slightly higher learning rate in the Haptics group (70\% vs 64\% in No-Haptics group) indicates that these subjects experienced a flatter learning curve, and that fewer trials were needed to reach the plateau. Figure 3 shows the percent time (normalized with session 1 as $100 \%$ ) in each session for the Haptics and No-haptics group. 


\section{Time to Task Completion}

Pair-wise comparison of individual learning curves showed a significantly faster time to task completion in the Haptics condition across the 18 training sessions, $t(179)=-2.1, p<.04$.

For every knot completed in training (the highest number of knots performed by a subject was 618 , average number was 200), subjects performed equally fast in both conditions, $F$ (1, $4165)=0.20, p<.653$ (Figure 4, only the first 182 knots are shown in the graph). The best trial in each session for subjects in the Haptics condition was significantly faster than that in the No-Haptics condition, $t(179)=-2.8, p<.006$ (Figure 5).

\section{Performance Variance}

The variance of task completion time per session in the No-Haptics condition was significantly higher than that in the Haptics condition, $F(1.341)=-8.31, p<.004$ (Figure 6). However, between subjects variance for each knot completed by at least two subjects during training in the No-Haptics condition was not significantly different than that in the Haptics condition, $F(1,611)=0.56, p<.457$ (Figure 7).

\section{Discussion}

Results in this study confirmed the hypotheses that haptic feedback can improve performance time during laparoscopic suturing and knot-tying training, but only for the first five hours of training. A standard measure of learning is the variability of performance during training; decreasing variability with trials indicates that the skill is being learned as performance becomes more consistent. In this experiment, haptic feedback allows trainees to perform more consistently in the initial stages of learning, with a slightly higher learning rate, and with a shorter learning curve. However, our learning curve results also suggest that the benefits of haptics in the latter stages of learning are minimal.

From Figure 5 and Figure 6, we observed that subjects took advantage of haptics at the very beginning of training, especially in the first two training sessions. In subsequent sessions, the learning curves of the Haptics and No-Haptics groups converge. This may be due to the fact that after initial familiarization with the instrumentation and task demands, where haptic feedback is beneficial, subjects concentrated on practicing the motor skills of suturing and knot-tying which require predominantly the coordination of hand gestures and tool movements. Suturing and knot-tying is a complex surgical task, requiring accurate positioning and controlling of instruments, needle, thread and tissue. Several key movements and gestures are involved in performing a successful suturing and knot-tying task, including positioning the needle to bite the tissue, rotating the needle driver to form the loop, and tightening the knot. As observed in this experiment, subjects seemed to rely mainly on visual feedback to position the needle to bite the tissue at the correct spot, and to manipulate the two needle drivers to form the loop. Furthermore, subjects in the Haptics group often relied on visual feedback to confirm that the knot was tight enough instead of relying on their haptic feedback alone. A previous study has suggested that haptic information perceived by the operator can disorient the learner when learning complex surgical tasks where gestures are more important [1]. Suturing and knot-tying is a task that is more about orientation, positioning, movements, and gestures of instruments and suturing materials, than it is about controlling the force application and differentiating tissue compliance. It seems that the performance of suturing and knot-tying were not affected dramatically by providing haptic feedback. Indeed, when inspecting suturing and knot-tying task separately in two phases in the Haptics condition, there were significant performance differences between sessions $1-3$ and the last session (session 18) in the suturing phase, while significant differences in performance were found between sessions 1-6 and the last 
session in the knot-tying phase. The suturing phase reached a performance plateau by the $4^{\text {th }}$ session, while the knot-tying phase reached the plateau by the $7^{\text {th }}$ session (Figure 8). This finding suggests that haptic feedback is more beneficial for suturing than for knot-tying, which requires more motor coordination than force control.

Although the benefits of haptics in the early stages of learning are subtle, those who learned with haptics were more consistent in performance, with a shorter learning curve. Conversely, trainees who trained without haptic feedback experienced dramatic decrease in performance consistency during the first several training sessions. There were also significant difference $(\mathrm{t}(179)=6.4, \mathrm{p}<.001)$ in the number of knots performed in each session by the two groups. Therefore, it might be worth the additional costs to equip laparoscopic surgery trainers with haptic feedback, provided that consistent performance is important during initial training, or that available time for training is limited (in light of the current constraints on training time and working hours for residents). With haptic feedback, the training simulator presents more realism that allows the trainees to be more comfortable initially, and results in faster performance stability in the learning curve. As the benefits of haptics depend on the nature of the task, the technical effort and financial investment of implementing haptic feedback functions into surgical simulators may not be justified by such subtle gains in learning. As observed in the current experiment, motor coordination demands are high in a laparoscopic suturing and knot-tying task, compared to previous studies on tissue probing tasks where force application control is more important.

One of the limitations of this study is that a post-training evaluation on an independent operating platform was not conducted. This would have enabled us to examine the effect of haptic feedback on skill retention and the transfer of skills into the operating room environment.

\section{Conclusion}

In general, haptic feedback enhances performance of laparoscopic surgery. It also benefits learning a complex surgical task, such as suturing and knot-tying, in the initial stage of training. Based on these results, haptic feedback may not be warranted in laparoscopic surgical trainers at all stages of training. The benefits of a shorter time to the first performance plateau and more consistent initial performance should be balanced with the cost of implementing haptic feedback in training simulators.

\section{Acknowledgments}

This work was supported in part by a National Science Foundation Career Award (IIS-0238284) and a Society of American Gastrointestinal Endoscopic Surgeons (SAGES) Research Grant Award.

\section{References}

1. Picod G, Jambon AC, Vinatier D, Dubois P. What can the operator actually feel when performing a laparoscopy? Surg Endosc. 2005; 19:95-100. [PubMed: 15772876]

2. Perreault JO, Cao CGL. Effects of Vision and Friction on Haptic Perception. Hum Factors. 2006; 48(3):574-86. [PubMed: 17063970]

3. Seymour NE, Gallagher AG, Roman SA, O’Brien MK, Bansal VK, Anderson DK, Satava RM. Virtual reality training improves operating room performance. Ann Surg. 2002; 236:458-464. [PubMed: 12368674]

4. Sutherland LM, Middleton PF, Anthony A, Hamdorf J, Cregan P, Scott D, Maddern GJ. Surgical simulation - A systematic review. Ann Surg. 2006; 243:291-300. [PubMed: 16495690] 
5. Grantcharov TP, Kristiansen VB, Bendix J, Bardram L, Rosenberg J, Funch-Jensen P. Randomized clinicl trial of virtual realtiy simulation for laparoscopic skills training. Brit J Surg. 2004; 91:146150. [PubMed: 14760660]

6. Lathan, CE.; Tracey, MR.; Sebrechts, MM.; Clawson, DM.; Higgins, GA. Using virtual environments as training simulators: measuring transfer. In: Stanney, KM., editor. Handbook of virtual environments: design, implementation, and applications. Lawrence Erlbaum Associates; Mahwah, NJ: 2002. p. 414-434.

7. Lamb GD. Tactile discrimination of textured surfaces: Psychophysical performance measurements in humans. J Physiol - London. 1983; 338:551-565. [PubMed: 6875970]

8. Lederman SJ. The perception of surface roughness by active and passive touch. B Psychonomic Soc. $1981 ; 18: 253-255$.

9. Lederman SJ. Tactual roughness perception: Spatial and temporal determinants. Can J Psychology. 1983; 37:498-511.

10. Srinivasan, M.; LaMotte, R. Tactual discrimination of softness: abilities and mechanisms. In: Franzen, O.; Johansson, R.; Terenius, L., editors. Somesthesis and the neurobiology of the somatosensory cortex. Birkhauser Verlag; Berlin: 1996. p. 123-136.

11. Lederman SJ, Klatzky RL. Haptic identification of common objects: Effects of constraining the manual exploration process. Percept Psychophys. 2004; 66:618-628. [PubMed: 15311661]

12. Klatzky RL, Lederman SJ, Hamilton CL, Grindley M, Swendsen RH. Feeling textures through a probe: Effects of probe and surface geometry and exploratory factors. Percept Psychophys. 2003; 65:613-631. [PubMed: 12812283]

13. Brydges R, Carnahan H, Dubrowski A. Surface exploration using laparoscopic surgical instruments: The perception of surface roughness. Ergonomics. 2005; 48:874-894. [PubMed: 16076743]

14. Botden SMBI, Torab F, Buzink SN, Jakimowicz JJ. The importance of haptic feedback in laparoscopic suturing training and the additive value of virtual reality simulation. Surg Endosc. 2007; 22:1214-1222. [PubMed: 17943369]

15. Kitagawa M, Dokko D, Okamura AM, Yuh DD. Effect of sensory substitution on suturemanipulation forces for robotic surgical systems. J Thorac Cardiov Sur. 2005; 129:151-158.

16. Moody L, Baber C, Arvanitis TN. Objective surgical performance evaluation based on haptic feedback. Stud Health Technol Inform. 2002; 85:304-310. [PubMed: 15458106]

17. Wagner, CR.; Stylopoulos, N.; Howe, RD. The Role of Force Feedback in Surgery: Analysis of Blunt Dissection. Proceedings of the 10th Symposium on Haptic Interfaces for Virtual Environment and Teleoperator Systsem (HAPTICS'02); 2002. p. 68-74.

18. Strom P, Hedman L, Sarna L, Kjellin A, Wredmark T, Fellander-Tsai L. Early exposure to haptic feedback enhances performance in surgical simulator training: a prospective randomized crossover study in surgical residents. Surg Endosc. 2006; 20:1383-1388. [PubMed: 16823652]

19. Smith CD, Farrell TM, McNatt SS, Metreveli RE. Assessing laparoscopic manipulative skills. Am J Surg. 2001; 181:547-550. [PubMed: 11513783]

20. O'Connor A, Schwaitzberg SD, Cao CGL. How much feedback is necessary for learning to suture? Surg Endosc. 2007; 22(7):1614-1619. [PubMed: 17973165]

21. Kothari SN, Kaplan BJ, DeMaria EJ, Broderick TJ, Merrell RC. Training in laparoscopic suturing skills using a new computer-based virtual reality simulator (MIST-VR) provides results comparable to those with an established pelvic trainer system. J Laparoendosc Adv S. 2002; 12:167-173.

22. Gallagher AG, Satava RM. Virtual reality as a metric for the assessment of laparoscopic psychomotor skills. Surg Endosc. 2002; 16:1746-1752. [PubMed: 12140641]

23. Pearson AM, Gallagher AG, Rosser JC, Satava RM. Evaluation of structured and quantitative training methods for teaching intracorporeal knot tying. Surg Endosc. 2002; 16:130-137. [PubMed: 11961623]

24. Champion, HR.; Higgins, GA. Meta-analysis and planning for SIMTRAUMA: medical simulation for combat trauma. U.S. Army Medical Research and Materiel Command; Fort Detrick, MD: 2000 . 
25. Cao CGL, Zhou M, Jones DB, Schwaitzberg SD. Can surgeons think and operate with haptics at the same time? J Gastrointest Surg. 2007; 11:1564-1569. [PubMed: 17710503]

26. Hassan I, Maschuw K, Rothmund M, Koller M, Gerdes B. Novices in surgery are the target group of a virtual reality training laboratory. Eur Surg Res. 2006; 38:109-113. [PubMed: 16699284]

27. Aggarwal R, Black SA, Hance JR, Darzi A, Cheshire NJW. Virtual reality simulation training can improve inexperienced surgeons' endovascular skills. Eur J Vasc Endovasc. 2006; 31:588-593.

28. Konz, S.; Johnson, S. Work Design Industrial Ergonomics Holcomb Hathaway. Scottsdale, AZ: 2000 . 


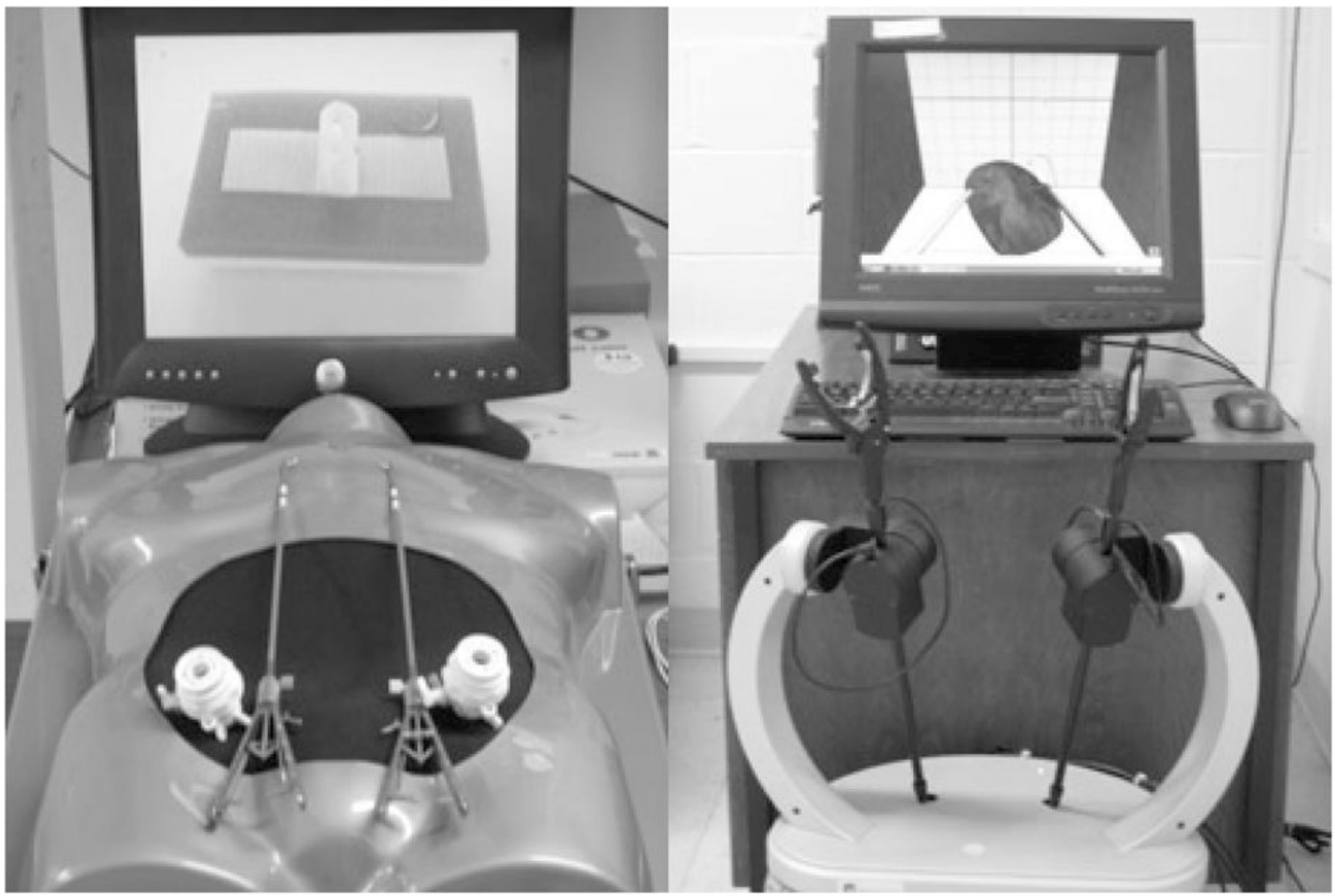

Figure 1.

Laparoscopic Surgical Simulators: ProMIS (left) and MIST-VR (right). 


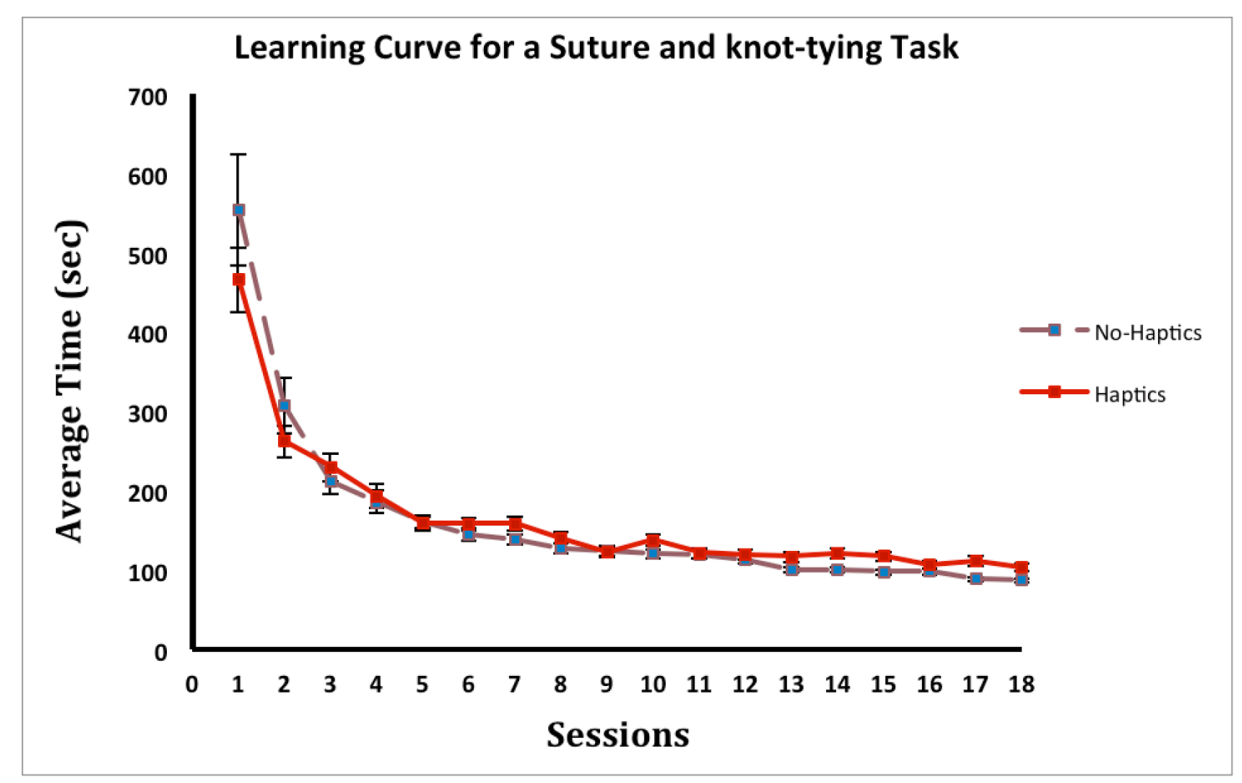

Figure 2.

The average time to task completion in each group per session. Error bars represent standard error. 


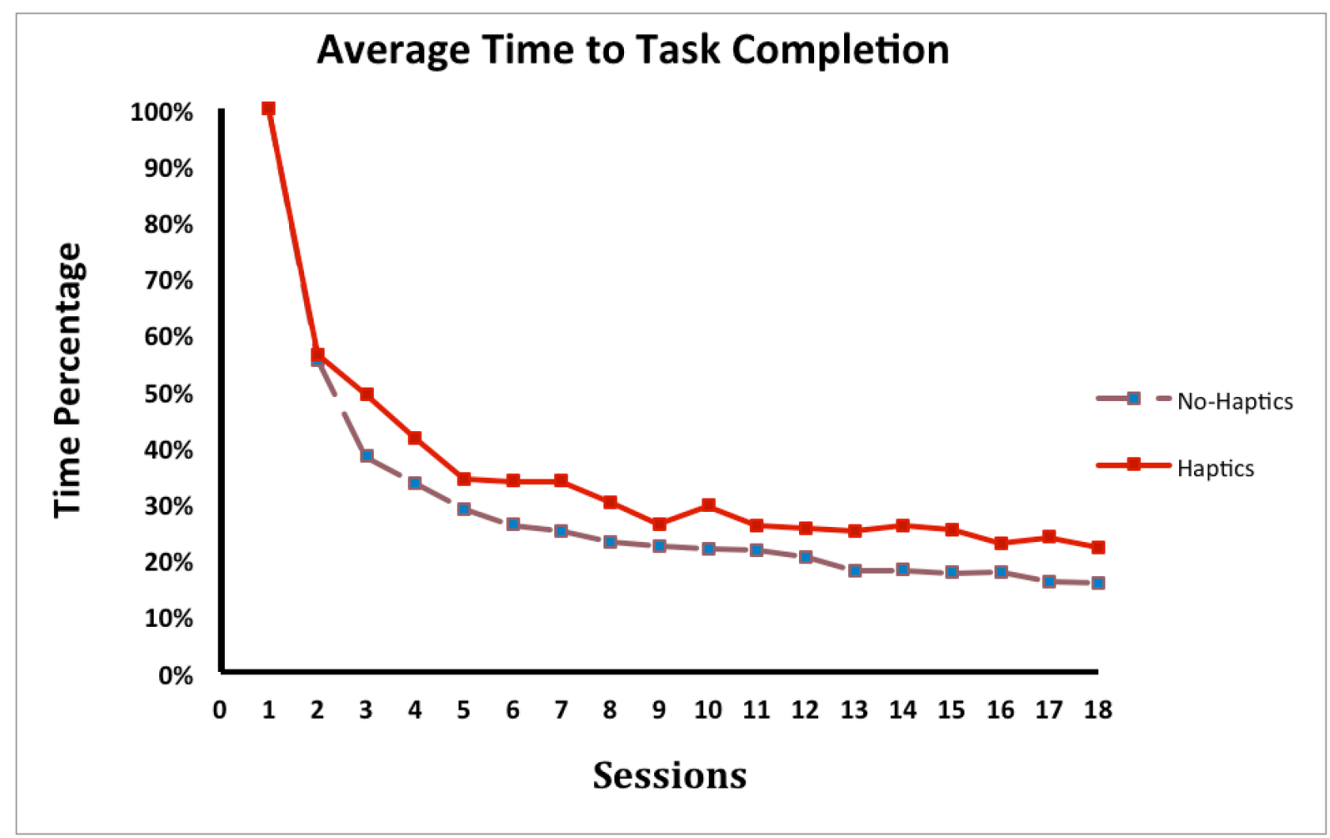

Figure 3.

The average time to task completion in percentage in each group. 


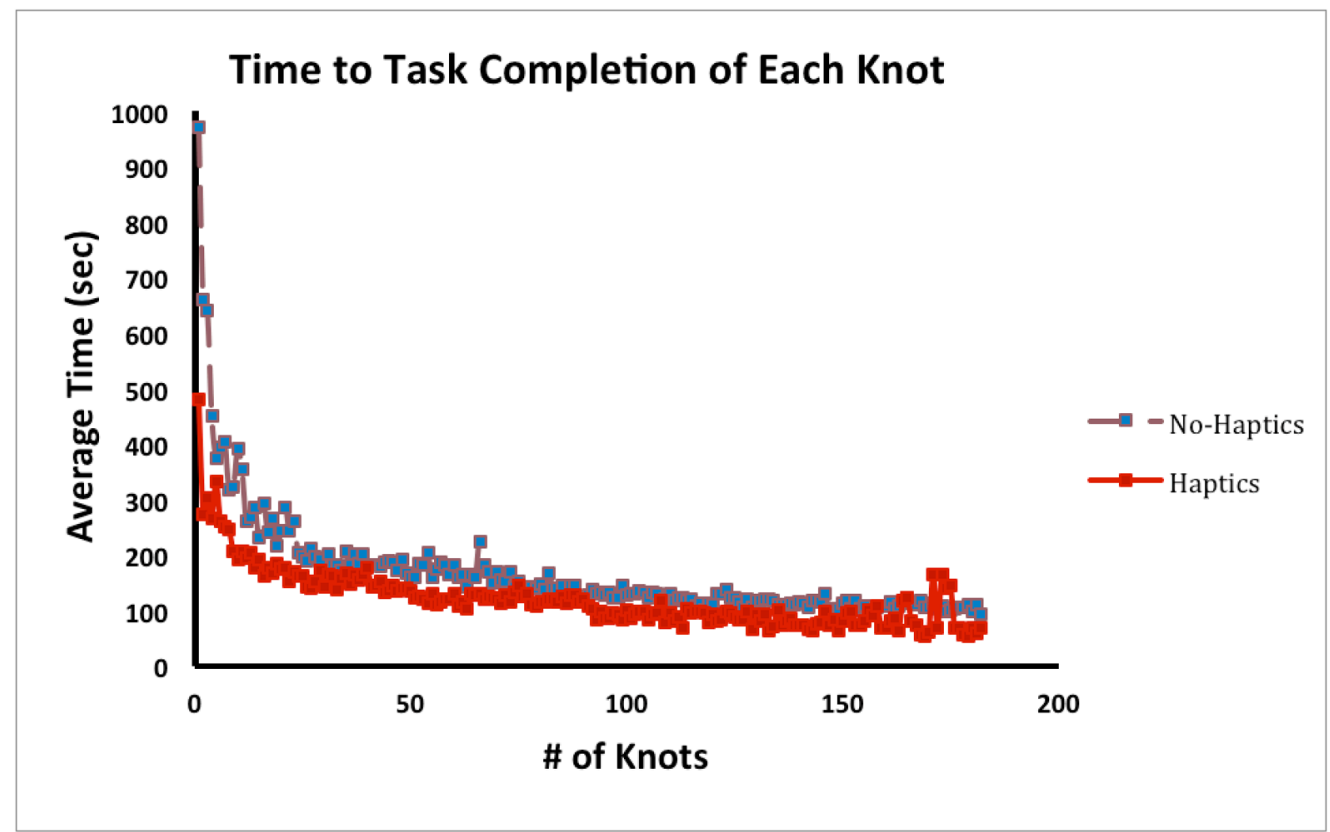

Figure 4.

The average time to task completion in each group for the first 182 knots. 


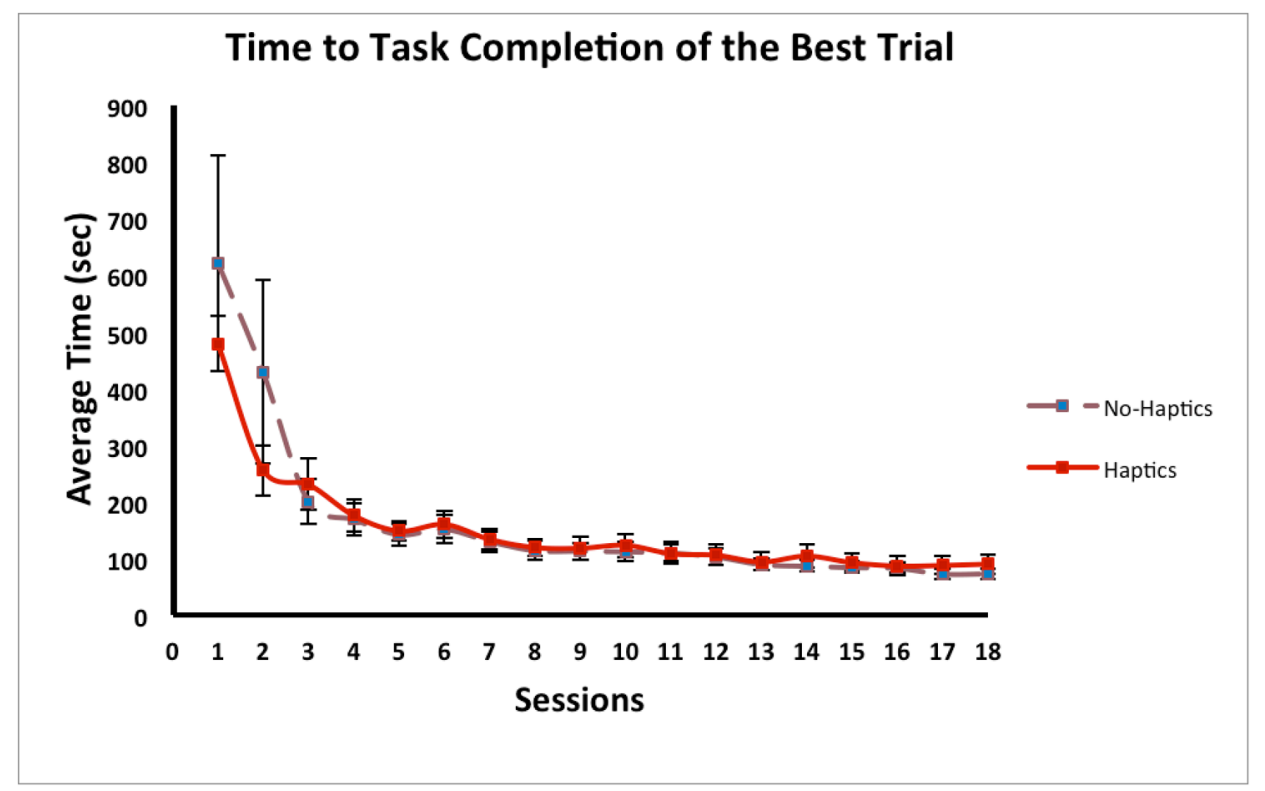

Figure 5.

The average time to ask completion in each group for the best trial of the session. Error bars represent standard error. 


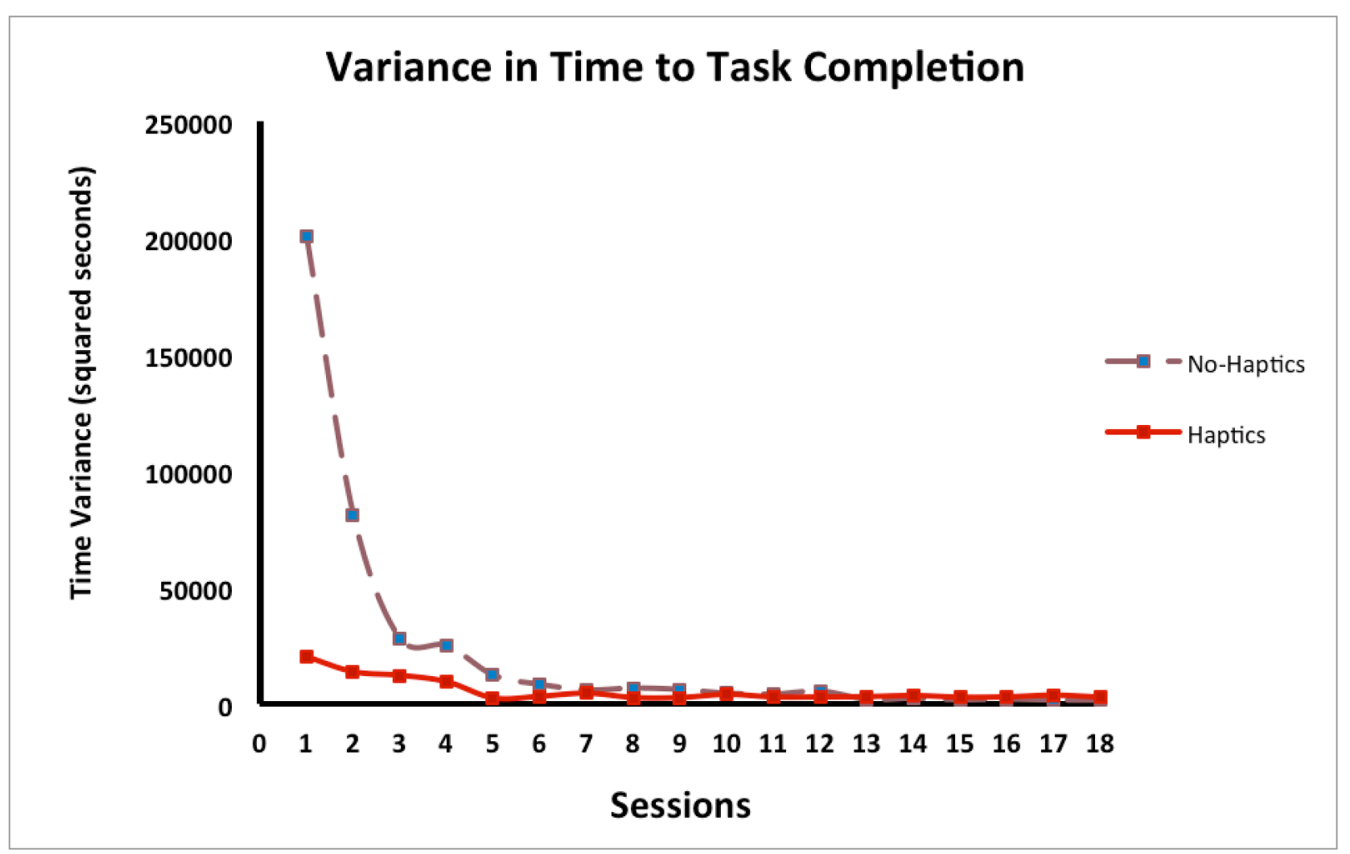

Figure 6.

The variance in time to task completion in each group per session. 


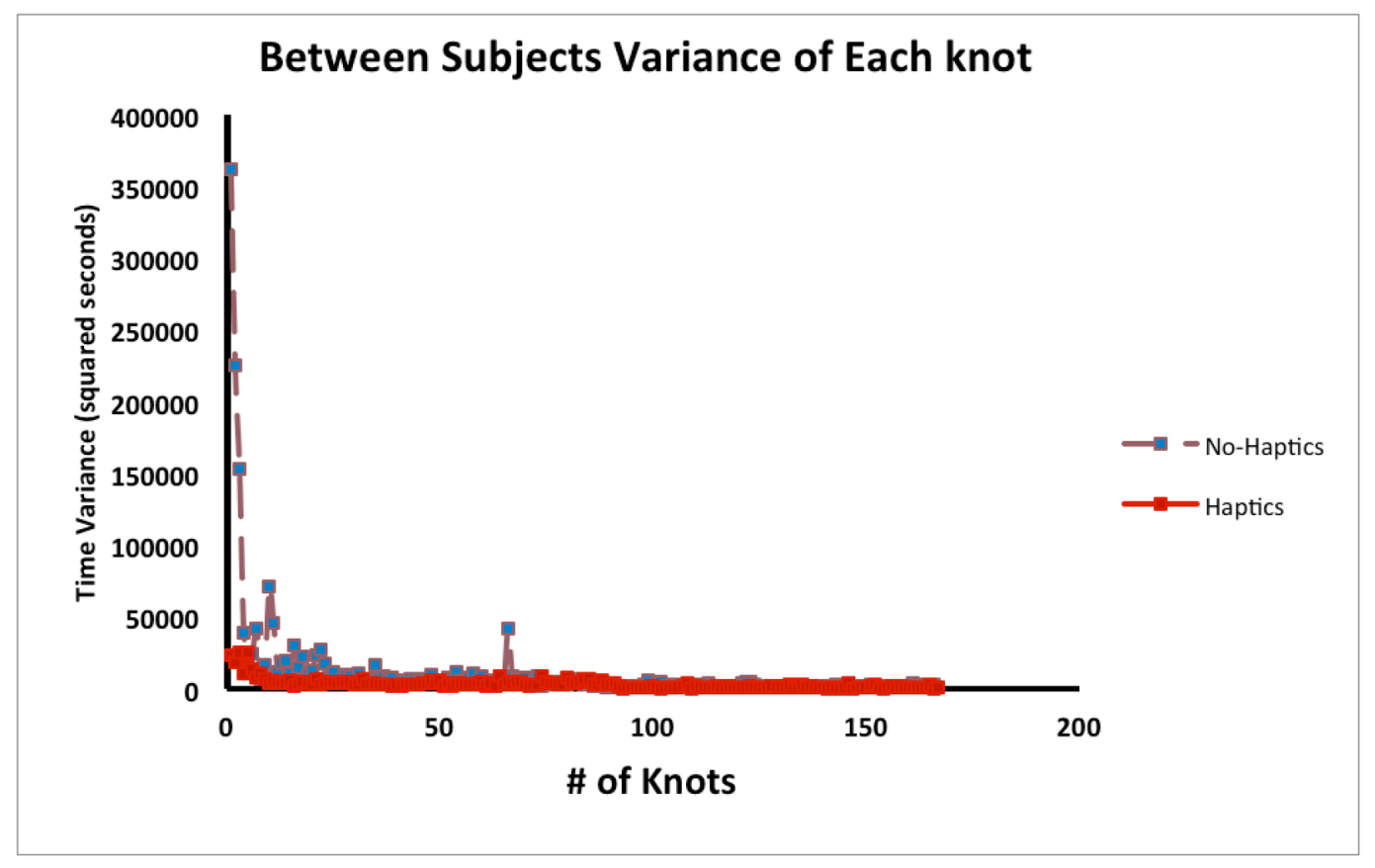

Figure 7.

The between subjects variance in time to task completion in each group for the first 182 knots. 


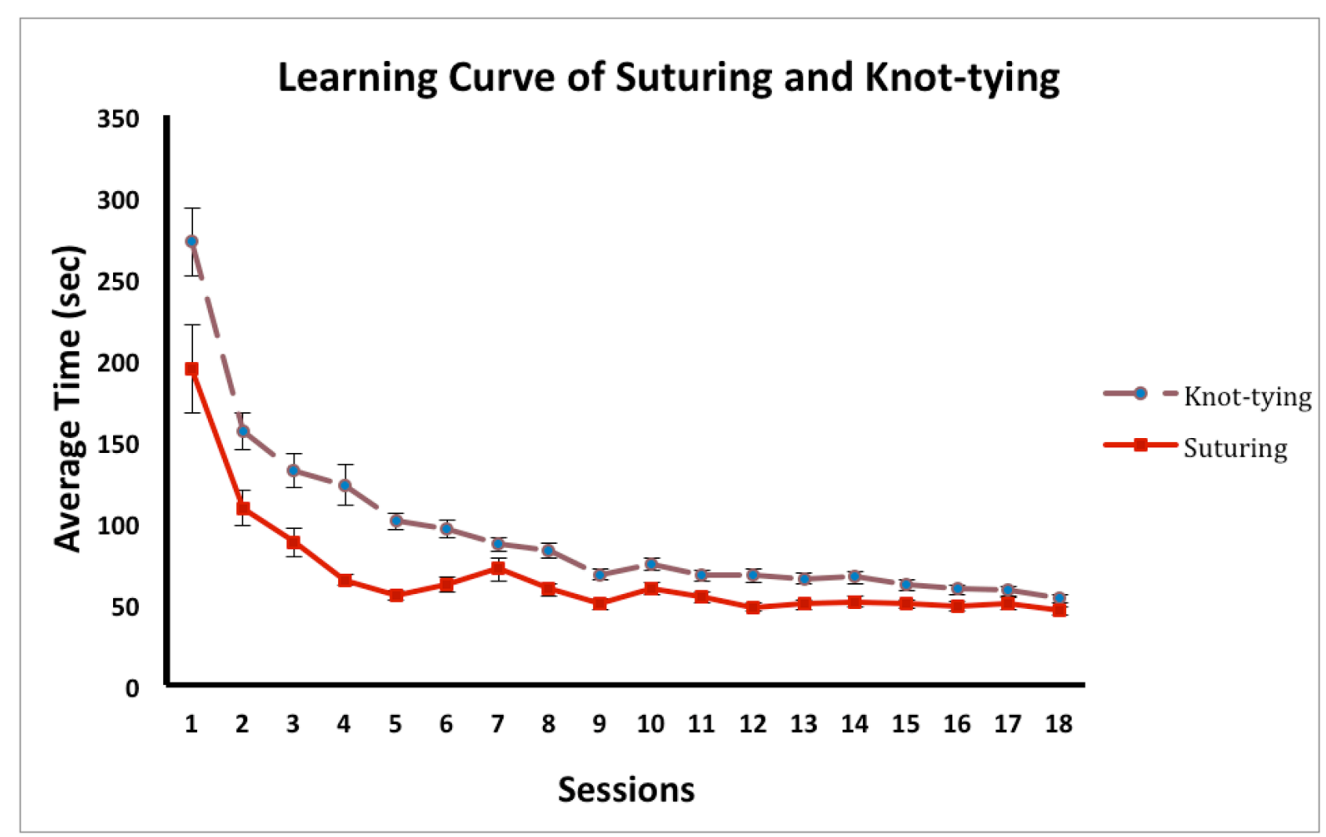

Figure 8.

The learning curves of suturing and knot-tying phases. Error bars represent standard errors. 


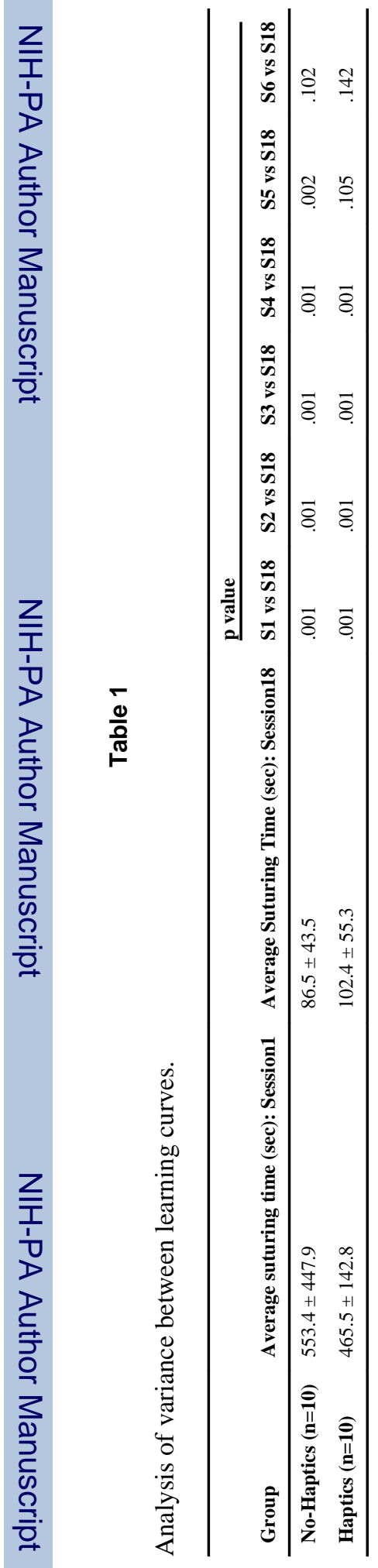

